\title{
A synthetic classic reinvented
}

The Diels-Alder cycloaddition, a classic synthetic organic reaction, is now being harnessed to label proteins.

Biologists never seem to lose their hunger for new chemical labeling strategies. Their ravenous need stems from the lack of a universal method of labeling biomolecules. Labeling methods can differ greatly, ranging from chemical to metabolic to genetic techniques, depending on whether the target biomolecule will be studied in an in vivo or an in vitro experiment. The types of labels and kinds of applications made possible by labeling methods vary even more widely, ranging from purification tags to fluorescent visualization labels to tags for spectroscopic identification and quantification.

Successful chemical labeling strategies usually stem from reactions that have been known and exploited for years in organic chemistry. A classic reaction that fits the bill is the Diels-Alder cycloaddition, which Herbert Waldmann and colleagues at the Max Planck Institute and the University of Dortmund in Germany have now applied as a new way to label proteins with fluorophores in vitro or immobilize proteins on surfaces.

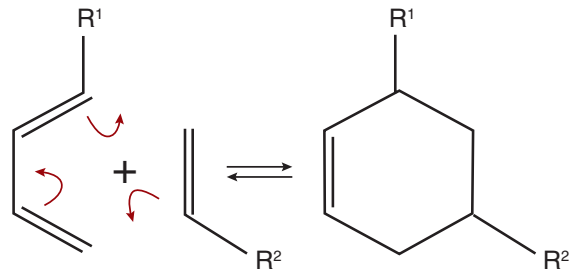

Figure 1 | The general Diels-Alder reaction. A diene (left) and a dienophile (middle) react to yield the cycloadduct, cyclohexene (right).

The Diels-Alder reaction involves a bondforming reaction between a diene and a dienophile, to yield cyclohexene (Fig. 1). Although the use of a cycloaddition reaction for a protein labeling strategy is somewhat unusual, it does have many desirable properties. In addition to being highly specific, "The Diels-Alder cycloaddition is accelerated in aqueous systems due to the hydrophobic effect," explains Waldmann. "It is compatible with many functional groups, and can operate at room temperature."

Waldmann and colleagues were particularly interested in protein arrays and found that there were surprisingly few bondforming reactions being harnessed for immobilizing proteins on surfaces. To use the Diels-Alder reaction for this purpose, they modify exposed lysine residues with a hexadiene. The array surface is functionalized with reactive maleimide (dienophile) molecules. "The ligation is a one-step process that does not require preactivation of a protein's C terminus or the array surface. It is operationally simple; you just mix and spot the compound," says Waldmann. They show that the method is also compatible with in vitro fluorescent labeling, especially when used in conjunction with expressed protein ligation for attaching the diene to the protein.

Thus, the Diels-Alder reaction is poised to become a useful-if unusual-new strategy, further expanding the repertoire of in vitro protein labeling methods. "The chemistry of protein synthesis is by far not yet developed to the extent that we can make any desired protein for a given application," says Waldmann. Clever chemists such as Waldmann will continue to fill the demand for new chemical labeling strategies as long as biologists need them.

\section{Allison Doerr}

\section{RESEARCH PAPERS}

Dantas de Araújo, A. et al. Diels-Alder ligation and surface immobilization of proteins. Angew. Chem. Int. Ed. 45, 296-301 (2006). 